Short communication

\title{
Responses to positive and negative smoking-related images: Effects of current smoking status and degree of smoking addiction
}

\author{
Laurence van Hanswijck de Jonge, Michael Gormley* \\ Department of Psychology, Aras an Phiarsaigh, University of Dublin, Trinity College, Dublin 2, Ireland
}

\begin{abstract}
The literature indicates that stimulant users of various dependency levels endorse and react differently to stimuli that portray the stimulant either positively or negatively, however these studies have not been extended to smoking. Here pictures are used to depict either positive or negative connotations of smoking. The current study concentrated on smokers with different levels of dependency. Seventy-three digital images were rated on both a smoking and emotional content scale. The pictures were rank ordered, yielding the 10 most positive and negative smoking-related pictures. Emotional content scores for these pictures were also recorded. Data from 148 subjects [light- $(n=28)$, heavy- $(n=17)$, ex- $(n=32)$ and never-smokers $(n=71)]$ were analysed. Using a mixed factorial ANOVA light- and heavy-smokers were found to score positive pictures significantly more positive than the never-smokers, they did not vary on negative or emotional ratings. Thus positive pictures did not distinguish heavy and light levels of substance use as suggested by the literature and this was not influenced by emotional content.
\end{abstract}

(C) 2005 Elsevier Ltd. All rights reserved.

Keywords: Smoking status; Emotional scale; Smoking scale; Positive pictures; Negative pictures

\footnotetext{
* Corresponding author. Tel.: +3531608 3903; fax: +35316712006.

E-mail address: Michael.Gormley@tcd.ie (M. Gormley).
} 


\section{Introduction}

In an attempt to understand the cognitive aspects of smoking behaviour, several studies have differentiated between stimuli that are perceived by smokers as depicting smoking in either a positive or negative light. These studies have found that positive, rather than negative, propositions/attributes related to smoking are more accessible in the smokers' memory. Although studies indicate that smokers and non-smokers will retrieve more antismoking 'schema' from memory when the speed of this retrieval is analysed, differences are obtained. Smokers, in comparison to non-smokers tend to produce most of the positive associations at earlier time intervals (Leung \& McCusker, 1999; Litz, Payne, \& Colletti, 1987). Furthermore studies from other addiction groups (e.g., alcoholism) indicate that these differences are not only to be found between addicts and non-addicts but also between heavy and light levels of the addiction. These studies suggest that individuals with heavy levels of an addiction have stronger associations with positive information and light levels with negative information (Armstrong, 2001; McCusker, 2001). This distinction has not been tested directly in smokers.

One difficulty with this smoking-related research is that a large proportion of the studies have been based on subjective creations of affective propositions over which the experimenter had little control or lengthy sentence stems that dictate the affect of the target word. The question remains whether the same distinctions are found when positive and negative smoking-related attributes are created more objectively. Words are also limited in the connotations they communicate. Images potentially portray a greater range of smokingrelated concepts with less ambiguity than single words. It is possible to capture pleasant or unpleasant smoking connotations in one single image, creating stimuli that could be used within broader research paradigms. Due to the possible presence of emotional content unrelated to smoking, such content would also have to be measured.

The aim of this research was two-fold. Firstly, to generate validated stimuli that depict either positive or negative aspects of smoking behaviour, while controlling for emotional content. Secondly, to determine whether heavy- and light-smokers vary on their ratings of these images replicating findings found in other addiction groups.

\section{Methods}

\subsection{Materials}

Seventy-three colour pictures were taken of smoking-related material in different situations. Picture size was adjusted to $6 \times 6 \mathrm{~cm}$ with a resolution of 28.35 pixels $/ \mathrm{cm}$ in Adobe Photoshop (Version 5.0 LE).

Each picture was presented with two rating scales presented below it. The first scale addressed the smoking content of the picture asking 'How does the smoking-related content of the picture make you feel?' Participants rated their responses on a 9-point scale from - 4 (very negative) to +4 (very positive). The second scale addressed the emotional content of the 
picture. Participants were asked 'Ignoring the smoking-related content rate the emotion in the picture'. A 7-point scale from 1 (very negative) to 7 (very positive) was used. The two scales were formatted differently to discourage participants from automatically entering the same response for both scales. The pictures, their rating scales, and a demographic and smokingrelated behaviour questions (Fagerstrom Tolerance Questionnaire; FTQ; Fagerstrom, 1978) were posted on a website.

\subsection{Picture categorization}

For each picture the mean score on both scales were calculated separately. The individual picture scores on both the smoking and emotion scales were converted to $z$-scores (using the mean and standard deviation for each scale). The pictures were then rank ordered according to their $z$-score. This enabled the identification of the 10 most positive and 10 most negative pictures in terms of smoking-related content. The mean $z$-scores from the emotional rating scale that corresponded with these 20 images were also recorded.

\subsection{Participants}

148 participants data were usable, 89 females (mean age $=28.9$ years, S.D. $=7.40$ ) and 59 males (mean age $=30.3$ years, S.D. $=5.87$ ). Participants were categorized into one of four groups, never-smokers $(n=71)$, ex-smokers $(n=32)$, light-smokers (smoking $<15$ cigarettes a day; $n=28, M=5.16$, S.D. $=3.79)$ and heavy-smokers (smoking $>14$ cigarettes a day; $n=17$, $M=20.44$, S.D. $=6.64)$. The light- $(M=104.69$, S.D. $=3.79)$ and heavy-smoking $(M=126.22$, S.D. $=83.80$ ) groups did not differ significantly in the length of time (measured in months), which they had been smoking for. FTQ scores $(t(48)=6.24, p<0.0001)$ indicated heavysmokers to be significantly more dependent $(M=6.05$, S.D. $=2.31)$ than the light-smokers $(M=3.19$, S.D. $=0.87)$.

\section{Positive picture ratings on two scales}

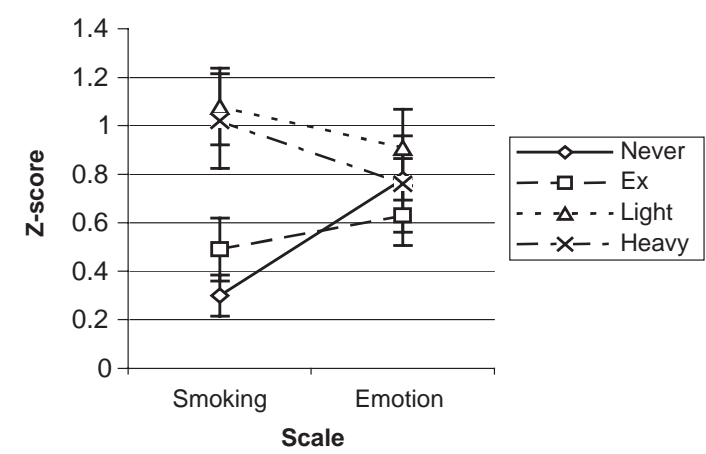

\section{Negative picture ratings on two scales}

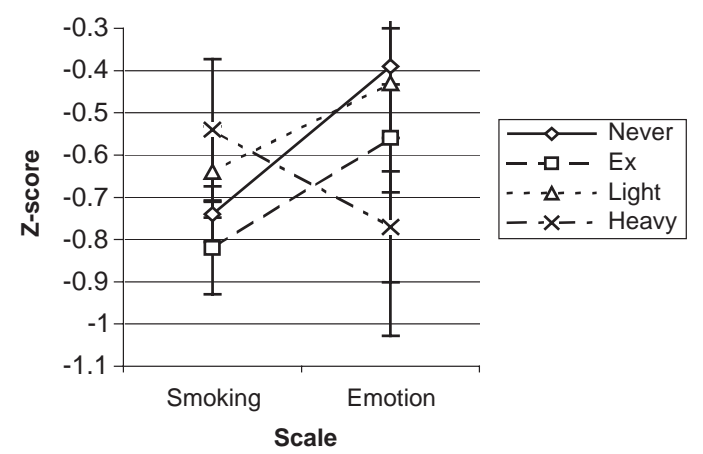

Fig. 1. Between group differences for ratings on the smoking and emotion scale for both the positively and negatively rated pictures (error bars indicate standard error). 


\section{Results}

A correlation was performed on the two scales to establish the amount of shared variance. With $r(144)=0.33, p<0.001$, the shared variance of 0.11 was relatively low indicating that the scales had a low shared variance.

A $4 \times 2 \times 2$ mixed factorial design was carried out with group as a between-subjects factor (never-smokers, ex-smokers, light-smokers and heavy-smokers) and both scale (emotion; smoking) and picture type (positive; negative) as within-subjects variables. This produced a significant three-way interaction between scale, picture and group $(F(3,141)=4.55, p<0.01)$. A simple main effects analysis of group indicated a significant effect of group for only the positive rated images on the smoking scale $(F(3,145)=9.78, p<0.001)$, as seen in Fig. 1 .

Lines are drawn between data points in Fig. 1 are to facilitate interpretation of interactions, not to extrapolate one from the other.

Post-hoc Tukey tests further showed these differences to be significant between the never and heavy-smoking groups $(p<0.01)$ and between the never- and light-smoking groups $(p<0.001)$. Both the light- $(M=1.08$, S.D. $=0.84)$ and heavy-smoking $(M=1.02$, S.D. $=0.82)$ group scored smoking-related content to be significantly more positive than the neversmoking group $(M=0.30$, S.D. $=0.71)$.

\section{Discussion}

The current results indicate that both the heavy- and light-smoking groups score positive images on the smoking scale significantly more positive than the never-smoking group. In contrast the four groups do not differ in their ratings of emotion in the pictures or on negative smoking-related images. Additionally, as the shared variance between the smoking scales and emotional scales was so low $(11 \%)$, this indicates that the two scales were measuring something different.

These data further support the idea that there are distinct differences between smoking and never-smoking groups and that these differences emerge only for stimuli which depict smoking in a positive light. Thus consistent with the previous research (Leung \& McCusker, 1999), smokers appear to be as aware of the negative connotations of smoking as nonsmokers and evaluate its negative depiction in a similar fashion. Since smokers and nonsmokers did not differ in how they rated the emotional content of the pictures, this can be ruled out as a source for the differences.

The current study does not support the results of the broader addiction related research, which found similar effects to be specific to the heavily dependent group but not the light dependent group (McCusker, 2001). More research needs to be conducted in order to identify whether this lack of differentiation could be due to underlying fundamental differences between levels of addiction in alcohol vs. nicotine dependence.

A further advantage to the creation of validated pictorial stimuli is that they can be used in a wider variety of research designs that aim to increase our understanding of smoking-related behaviour and cognitions. 


\section{References}

Adobe Photoshop version 5.0 LE [computer software]. (2003). Adobe systems incorporated, San Jose, California 95110-2740, USA.

Armstrong, C. (2001). Automatic vs. non-automatic cognitive biases in social and dependent drinkers (Doctoral dissertation, Queens University Belfast UK. Dissertation Abstract International, DAI-C 62/04, p. 645.

Fagerstrom, K. O. (1978). Measuring degree of physical dependence to tobacco smoking with reference to individualisation of treatment. Addiction Behaviour, 3, 235-241.

Leung, K. S., \& McCusker, C. G. (1999). Accessibility and availability of smoking-related associations in smokers. Addiction Research, 7, 213-226.

Litz, B. T., Payne, T. J., \& Colletti, G. (1987). Schematic processing of smoking information by smokers and never-smokers. Cognitive Therapy and Research, 11, 301-313.

McCusker, C. (2001). Cognitive biases and addiction: an evolution in theory and method. Addiction, 96, 47-56. 